\title{
Estratégias de Enfrentamento de Enfermeiros frente ao Paciente Oncológico Pediátrico
}

\author{
Coping Strategies of Nurses toward Patients in Pediatric Oncology \\ Estrategias de Enfrentamiento de los Enfermeros hacia al Paciente Oncológico \\ Pediátrico
}

\author{
Maria Gizelda Gomes Lages', Maria Amélia de Oliveira Costa², Thayson Rodrigues Lopes ${ }^{3}$, Fráyla Cristinne Sousa de Amorim \\ Ari Pereira de Araujo Neto ${ }^{5}$, Ianny Raquel Dantas Nascimento, Charllyton Luis Sena da Costa ${ }^{7}$
}

\section{Resumo}

Introduçáo: $\mathrm{Na}$ dificuldade de cuidar de crianças com câncer, pode-se utilizar estratégias de enfrentamento, entendidas como um conjunto de respostas comportamentais do indivíduo, diante de situação estressora e na tentativa de adaptar-se ao evento estressor. Objetivos: Avaliar as estratégias de coping de enfermeiros frente ao paciente oncológico pediátrico; determinar a quantidade de enfermeiros que utilizam coping no seu dia a dia ao cuidar de pacientes oncológicos pediátricos, identificar as estratégias coping e as situaçôes de enfrentamento dos enfermeiros ao cuidar dos pacientes oncológicos pediátricos. Método: A pesquisa foi de natureza quantitativa, descritiva e exploratória. A coleta de dados deu-se através da aplicação do Inventário de Estratégias de Coping, no hospital referenciado para o tratamento de doenças oncológicas, Teresina (PI) de março a setembro de 2010. A população-alvo do estudo foi constituída por todos os enfermeiros que já trabalharam ou trabalham na área de oncologia pediátrica do referido hospital; de um total de 20 enfermeiros, 12 aceitaram participar. Resultados: Os resultados demonstram que a totalidade da população entrevistada utilizou estratégias de coping e a situação estressante mais citada foi a fase terminal, sendo a estratégia de enfrentamento de resoluçáo de problemas a mais utilizada por 11 enfermeiros $(93,8)$ e a menos, fuga-esquiva utilizada por nove enfermeiros (75\%). Conclusáo: A menor utilização de estratégias negativas demonstra que os enfermeiros se preocupam, se envolvem, e não negam suas responsabilidades no processo em que se encontram. Os Enfermeiros pesquisados utilizam predominantemente estratégias positivas, embora ainda recorram a meios que não ajudam no enfrentamento.

Palavras-chave: Enfermagem Pediátrica; Enfermagem Oncológica; Relações Enfermeiro-Paciente; Adaptação Psicológica; Criança

\footnotetext{
${ }^{1}$ Enfermeira. Formada pela Faculdade Integral Diferencial (FACID). Teresina (PI), Brasil. E-mail: gizelda_lages@hotmail.com.

${ }^{2}$ Orientadora. Docente da FACID. Mestre pela Universidade Federal do Piauí (UFPI). Teresina (PI), Brasil. E-mail: ameliao.costa@hotmail.com.

${ }^{3}$ Mestrando em Biotecnologia pela UFPI. Químico. Formado pela Universidade Estadual do Piauí (UESPI). Parnaíba (PI), Brasil. E-mail: thayson. rodrigues@hotmail.com.

${ }^{4}$ Acadêmica de Fisioterapia da UFPI. Parnaíba (PI), Brasil. E-mail: frayla_cristinne@yahoo.com.br.

${ }^{5}$ Acadêmico de Biomedicina da UFPI. Parnaíba (PI), Brasil. E-mail: arineto_ufpi@hotmail.com.

${ }^{6}$ Estudante de Enfermagem da NOVAFAPI. Teresina (PI), Brasil. E-mail: iannyr@hotmail.com.

${ }^{7}$ Docente da FACID. Doutorando pela Rede Nordeste de Biotecnologia (RENORBIO). Teresina (PI), Brasil. E-mail:farmácia@facid.com.br. Trabalho realizado no Hospital São Marcos e apresentado parcialmente no $13^{\circ} \mathrm{CBCEnf}$ em Natal.

Enderę̧o para correspondência: Maria Gizelda Gomes Lages. Rua Marechal Piares Ferreira, S/N, Centro. Barras (PI), Brasil. CEP: 64100-000.
} 


\section{INTRODUÇÃO}

Pela complexidade do câncer, a assistência à criança acometida por essa doença deve ser bastante abrangente, através de um cuidar holístico, observando tanto os fatores físicos quanto psicológicos, já que essa patologia é uma das que mais causam dor, sofrimento, medo, ansiedade e estresse ao paciente, família e aos profissionais que cuidam de tais crianças.

Ao longo do processo terapêutico, a enfermagem tem uma maior possibilidade de contato com o paciente, por não limitar suas açôes apenas para procedimentos meramente técnicos, pois aliam-se às diversas características relativas ao ser humano, dessa forma privilegiam os aspectos sociopsicoespirituais ${ }^{1}$.

As neoplasias possuem localizações e aspectos clínicos variados e não possuem sintomas ou sinais específicos, podendo ser detectados em vários estágios de evolução histopatológica e clínica. Por isso que, na maioria das vezes, existe a dificuldade do seu diagnóstico e a afirmação de que a suspeita de câncer pode surgir diante dos mais variados sintomas ${ }^{2}$.

Os novos tratamentos apresentam dois principais objetivos: aumentar a sobrevida, de modo a minimizar os efeitos tardios do tratamento; e reconduzir a criança na sociedade com qualidade de vida. Dessa forma, contam com três modalidades: quimioterápica, radioterápica e cirúrgica, incluindo, nesse primeiro tipo, as drogas citostáticas comumente denominadas de quimioterapia antineoplásica. Em alguns casos, utiliza-se uma modalidade terapêutica separadamente, e, em outros, pode existir uma combinação de duas ou até das três modalidades ${ }^{3}$.

Com relação ao câncer, os profissionais enfermeiros devem atuar nas ações de prevenção e controle, eles devem cuidar desses pacientes que possuem câncer tanto na avaliação diagnóstica como no tratamento, reabilitação e atendimento aos familiares. Além dessas, eles desenvolvem educaçáo em saúde, açóes integradas com outros profissionais, apoiam medidas legislativas e identificam fatores de risco ocupacional na prática da assistência ao paciente oncológico e sua família ${ }^{4}$.

Logo, o exercício profissional no âmbito hospitalar é marcado por múltiplas exigências, o setor de oncologia porta uma particularidade que são as representações do câncer fortemente associadas à ideia de morte . Aqueles que lidam com pacientes oncológicos estão mais intensamente mobilizados visto que, no câncer, a dimensão real desses acontecimentos é valorizada e requintada pela força simbólica que a doença carrega ${ }^{6}$.

O estresse causado pelo exercício profissional denota o estado gerado pela percepção de estímulos que provocam excitação emocional e, ao perturbarem a homeostasia, disparam um processo de adaptação caracterizado entre outras alteraçôes, pelo aumento de secreção de adrenalina produzindo diversas manifestaçôes sistêmicas com distúrbios fisiológicos e psicológicos. $\mathrm{O}$ termo estressor por sua vez define o evento ou estímulo que provoca ou conduz ao estresse?

O enfermeiro é o profissional que cuida do paciente de forma holística, sempre ao seu lado, prestando-lhe cuidados diretos e através dessa relação desenvolvem um sentimento afetivo. Essa técnica desencadeia proximidade do profissional que cuida e de quem está sendo cuidado, ao tornar-se diferente e preferida. É considerado como uma base de segurança e quando por algum motivo é interrompido, como o que acontece com a morte, gera sofrimento e sentimento de perda, ou seja, provoca o luto que é uma resposta esperada frente à separação ${ }^{8}$.

$\mathrm{Na}$ dificuldade de cuidar de crianças com câncer, podem ser usadas estratégias de enfrentamento, processo que pode ser definido, então, como um conjunto de respostas comportamentais que o indivíduo, diante de uma situação de estresse, emite para modificar o ambiente na tentativa de adaptar-se da melhor forma possível ao evento estressor, de maneira a reduzir ou minimizar seu caráter aversivo. Tais estratégias são aprendidas e mantidas ou não no decorrer da vida de cada indivíduo, o que vai depender dos esquemas de reforçamento a que cada um foi submetido durante sua história ${ }^{9}$. Não existe uma tradução para o português de coping, mas muitos autores utilizam a palavra enfrentamento ${ }^{10}$.

O modelo de coping de Folkman e Lazarus é conceituado de quatro formas básicas: o primeiro está relacionado com o processo que ocorre entre indivíduo e ambiente, o segundo com a função que o coping tem em relação a administrar a situação estressora, o terceiro diz respeito à percepção, interpretação e entendimento da situação avaliada como estressante e, por fim, o quarto conceito que constitui atividades de esforços cognitivos e comportamentais para administrar o evento estressor, ou seja, o indivíduo deve perceber o evento, analisar as opçōes e só assim utilizar a estratégia mais favorável para aquele momento ${ }^{11}$.

Folkman e Lazarus (1980) enfatizam o papel assumido pelas estratégias de coping, mostrando que essas estratégias podem mudar de um momento para outro, durante as etapas de uma situação estressante. De acordo com essas mudanças nas reaçóes individuais, não existe a possibilidade de se tentar predizer respostas situacionais a partir do estilo específico de coping de uma pessoa ${ }^{12}$.

De acordo com esses, as estratégias dependem muito da sua funçáo e podem ser de dois tipos. O coping focalizado na emoção, que é tido como um esforço para controlar o estado emocional e está associado ao estresse, ou a resultado de situaçóes estressantes. Essa forma tem como objetivo mudar o emocional do indivíduo que sofreu o estresse, diminuindo a sensação física desagradável de um estado de estresse ${ }^{12}$. Já no coping focalizado no problema, a 
preocupação maior está na tentativa de mudar a situação estressante; ao definir o problema, deve-se enumerar as opçóes, comparando-as em termos de custo e benefício e escolher uma atitude. Esse é considerado mais adaptativo, pois apresenta a capacidade de diminuir ou eliminar a fonte de estresse. A ação de coping pode está direcionada ao ambiente (fonte externa de estresse), ou à própria pessoa (fonte interna de estresse), sendo necessária a modificação dos estados motivacionais e cognitivos do indivíduo ${ }^{13}$.

A justificativa desse estudo é dada pela importância que é direcionada ao câncer, pois é uma patologia que devasta tanto o físico, social, quanto o psicológico, o que afeta o paciente, família e o enfermeiro, sendo esses por prestarem algum tipo de cuidado a esse paciente, por isso se faz importante o estudo das estratégias de coping utilizadas pelo enfermeiro como forma de aliviar o estresse do dia a dia ao cuidar de paciente oncológico pediátrico.

Este estudo tem como relevância avaliar as estratégias de coping dos enfermeiros; pois, a partir do conhecimento da estratégia utilizada, poderá adequar suas açóes às suas necessidades e assim melhorar a qualidade da assistência prestada ao paciente oncológico pediátrico e da família, através do bem-estar do enfermeiro envolvido nesse processo de cuidar, além de subsidiar o serviço no próprio hospital, pois esse poderá realizar uma melhor capacitação desses profissionais e assim melhorar o atendimento e rendimento de cada profissional.

O problema de pesquisa deste trabalho foi verificar se os enfermeiros utilizam estratégias de coping ao cuidar de pacientes oncológicos pediátricos, tendo como hipótese que a utilização dessas estratégias de coping por enfermeiros interfere positivamente no cuidado ao paciente oncológico pediátrico.

O objetivo geral foi avaliar as estratégias de coping de enfermeiros frente ao paciente oncológico pediátrico; e os objetivos específicos foram: determinar a quantidade de enfermeiros que utilizam coping no seu dia a dia ao cuidar de pacientes oncológicos pediátricos, identificar as estratégias coping e as situaçôes de enfrentamento dos enfermeiros ao cuidar dos pacientes oncológicos pediátricos.

\section{MÉTODO}

Esta é uma pesquisa de natureza quantitativa definida como o desenvolvimento de conhecimento, ou seja, raciocínio de causa e efeito, redução de variáveis específicas, hipóteses e questôes, uso de mensuração, observação e teste de teorias. Neste tipo de estudo, o survey é a melhor expressão de instrumento, ainda fazendo-se uso da pesquisa descritiva e exploratória com a finalidade de melhor caracterizar o estudo aqui utilizado.

Foi realizada em um hospital filantrópico referenciado para tratamento de doenças oncológicas na cidade de
Teresina (PI) cujos quadros funcionais contemplam a população pesquisada.

A populaçáo-alvo do estudo foi constituída por todos os enfermeiros que já trabalharam ou trabalham na área de oncologia pediátrica do referido hospital, sendo que, do total de 20 enfermeiros, a amostra final totalizou apenas 12 , que se enquadraram no critério de inclusão (ser enfermeiro do hospital, ter vivenciado uma situação de estresse e aceitar participar da pesquisa).

Foram incluídos no estudo enfermeiros do hospital que já haviam vivenciado algum tipo de estresse ao cuidar de paciente oncológico pediátrico, uma vez que foram avaliadas as estratégias que os sujeitos utilizaram para o enfrentamento de certas situações estressantes.

$\mathrm{O}$ instrumento utilizado nesta pesquisa foi o Inventário de Estratégias de Coping de Lazarus e Folkman (1984), utilizado para avaliar como os enfermeiros que lidam com as demandas internas ou externas de um evento estressante. Esse instrumento é composto por 66 itens que incluem pensamentos e açôes, e cuja intensidade é medida por meio de uma escala tipo Likert de três pontos que varia de 0 (não utiliza) a 3 (utiliza em grande quantidade). Os itens que compóem o inventário são divididos em oito fatores classificatórios, que foram reorganizados e mantidos por Savóia, Santana e Mejias ${ }^{14}$ após a verificação da confiabilidade e validade do instrumento à realidade brasileira. São eles: confronto (itens 7, 17, 28, 34, 40, 47), afastamento (itens $6,10,13,16,21,41,44$ ), autocontrole (itens $14,15,35,43,54$ ), suporte social (itens 8, 18, 22, $31,42,45$ ), aceitação de responsabilidade (itens 9, 25, $29,48,51,52,62$ ), fuga-esquiva (itens 58,59 ), resolução de problemas (itens $1,26,46,49)$ e reavaliação positiva (itens 20, 23, 30, 36, 38, 39, 56, 60, 63).

Inicialmente, a pesquisa foi aprovada pelo Comitê de Ética da Faculdade Integral Diferencial (FACID) com protocolo de número 388/09. Em Seguida, foi realizado contato com o local da pesquisa referenciado para tratamento de doenças oncológicas na cidade de Teresina (PI), a fim de se obter autorização para a realização da pesquisa entre os enfermeiros, por meio de uma carta de apresentação e de autorização; posteriormente, foi estabelecido contato com os sujeitos a serem avaliados. Foi entregue, para cada sujeito que aceitou participar, o Termo de Consentimento Livre e Esclarecido (TCLE), explicitando os objetivos da pesquisa e os procedimentos do estudo, junto com o Inventário de Estratégias de Coping. A aplicação do Inventário de Estratégias de Coping ocorreu no hospital, entre os meses de março a setembro de 2010, onde foram entregues para o preenchimento.

Os dados coletados foram analisados pelo pacote estatístico SPSSWIN versão 17.0 com vistas a identificar grupos de sujeitos e suas estratégias de coping. A análise utilizou-se de procedimentos estatísticos descritivos como frequência relativa e absoluta, de forma a criar um 
quadro descritivo dos procedimentos de enfrentamento mais utilizados pelos sujeitos da pesquisa. A análise dos dados foi realizada com base em bibliografia relacionada a: estratégias de coping e sintomas de estresse, e fatores estressantes e estratégias de enfrentamento utilizadas por enfermeiros de oncologia.

\section{RESULTADOS E DISCUSSÃO}

Entre diversas estratégias de enfrentamento existentes, o que determina a sua escolha é a natureza do estressor, as circunstâncias em que ele se reproduz, a história prévia de confronto e o próprio estilo que caracteriza o sujeito, ou seja, como ele enfrentou as situaçóes anteriores. Há dois tipos básicos de indivíduos, aqueles que apresentam tendência evitadora e minimizadora e aqueles com estilo afrontativo ou vigilante ${ }^{14}$.

Alguns autores complementam que enfrentamento está relacionado ao desenvolvimento de habilidades dos indivíduos para controlarem e adaptarem-se às situaçóes de estresse, ou seja, é uma resposta ao estresse. E que as pessoas possuem diferentes níveis de tolerância a situaçóes estressantes. Dessa forma, enquanto algumas são afetadas pela menor mudança em sua vida, outras são afetadas apenas por estressores de maior intensidade ou quando a exposiçẫo a estes é muito prolongada ${ }^{15}$.

Nota-se que, cada vez mais, o enfermeiro está sendo chamado a se preparar para enfrentar a problemática do doente portador de doença crônica degenerativa ou fora de possibilidades terapêuticas, uma vez que este o coloca face a face com as frustraçôes de um trabalho com poucos retornos gratificantes, no qual se torna necessário entender as construçóes culturais cumulativas que medeiam o significado imputado às emoçôes no dia a dia do seu trabalho ${ }^{16}$.

Com isso, o entendimento das estratégias irá ajudar em uma melhor adaptaçáo aos fatos estressantes, pois com esse conhecimento será mais fácil a sua mudança, já que as estratégias estão relacionadas a fatores situacionais, podendo mudar de momento para momento durante cada situação de estresse, significando que uma estratégia que foi adaptativa em uma situação pode ser totalmente prejudicial em outra.

$\mathrm{Na}$ Tabela 1, que apresenta a frequência de utilização para cada estratégia, é possível visualizar que, apesar de a estratégia de resolução de problemas ter sido utilizada por um maior número de participantes, a estratégia de reavaliação positiva foi empregada com mais frequência.

Os resultados desta pesquisa mostram que todos os oito fatores propostos por Lazarus e Folkman (1984) foram evidenciados e que todos os sujeitos envolvidos na pesquisa (100\%) utilizaram dessas estratégias, o que corroboram pesquisas anteriores que revelam ligação entre as diferentes estratégias, podendo apresentar ou não o predomínio de uma determinada estratégia. Esta pesquisa mostra a predominância de três: resolução de problemas $(93,8 \%)$, reavaliaçáo positiva $(86,1 \%)$, suporte social $(84,8 \%)$. Ao escolher a situaçáo de estresse para proceder ao preenchimento do Inventário de Coping, verificou-se que a fase terminal do paciente oncológico foi citada em $50 \%$ pelos enfermeiros, esses resultados conferem com os achados de Rodrigues e Chaves ${ }^{17}$ que avaliaram os fatores estressantes e as estratégias de coping de enfermeiros envolvidos no tratamento de pacientes oncológicos.

Com tudo isso, o enfermeiro está próximo dos pacientes nos momentos difíceis, por isso é solicitado por tais quando necessitam de esclarecimentos, ou de cuidados imediatos. Assim esse profissional tem que estar preparado para conviver todos os dias com o sofrimento, com a angústia e com os temores que podem surgir em diversas situaçóes que envolvem esse cuidar ${ }^{8}$.

Nesta pesquisa, a estratégia de confronto (Tabela 1) foi a menos utilizada, pois $44,4 \%$ da população pesquisada não utilizaram esta estratégia e 6,9\% a usaram em grande quantidade. Resultados semelhantes foram encontrados em outros estudos ${ }^{18-21}$, cabendo salientar que, com esses resultados, a maioria buscou evitar comportamentos com intençốes de confronto diante do ocorrido, pois tais

Tabela 1. Distribuição percentual da utilização das Estratégias de Coping pelos sujeitos pesquisados no município de Teresina (PI, 2010)

\begin{tabular}{l|l|l|l|l}
\hline Fatores & Não usou & Pouco & Bastante & $\begin{array}{l}\text { Grande } \\
\text { quantidade }\end{array}$ \\
\hline Confronto & 44,4 & 34,7 & 13,9 & 6,9 \\
\hline Afastamento & 35,7 & 33,3 & 22,6 & 8,3 \\
\hline Autocontrole & 18,3 & 18,3 & 36,7 & 26,7 \\
\hline Suporte social & 15,3 & 16,7 & 40,3 & 27,8 \\
\hline Aceitação de responsabilidade & 17,9 & 25,0 & 38,1 & 19,0 \\
\hline Fuga e esquiva & 25,0 & 29,2 & 37,5 & 8,3 \\
\hline Resolução de problemas & 6,3 & 6,3 & 52,1 & 35,4 \\
\hline Reavaliação positiva & 13,9 & 13,0 & 33,3 & 39,8 \\
\hline
\end{tabular}


estratégias correspondem às formas ofensivas com esforços agressivos, podendo, muitas vezes, apresentar grau de hostilidade e risco envolvido para o enfrentamento da situação, isto é, são estratégias nas quais a pessoa apresenta uma atitude ativa em relação ao estressor ${ }^{22}$. Nem sempre as estratégias envolvidas no confronto com a situação levam a resultados positivos, como o fato de descontar a raiva em outras pessoas, fugir das pessoas em geral e extravasar seus sentimentos.

Diferentemente das estratégias de confronto, o afastamento corresponde a estratégias defensivas, centradas na emoção, nas quais o indivíduo evita confrontar-se com a ameaça, não modificando a situação, esforçando-se para o desprendimento e diminuição da intensidade da situação $^{22,10}$. O índice encontrado (Tabela 1) mostra que 35,7\% dos participantes não utilizaram essa estratégia e $8,3 \%$ a utilizaram em grande quantidade. Este resultado demonstra que os enfermeiros não negaram ou recusaram ter contato com o fato desestruturante. Outras pesquisas também demonstram tais resultados ${ }^{18,20-21}$, diferentemente de outro estudo que avaliou as estratégias de coping na adolescência, no qual os adolescentes estão utilizando estratégias de escapar do problema, ou seja, estáo negando enfrentar o fato estressante, diferente também do estudo em questáo ${ }^{10}$.

O autocontrole (Tabela 1) não foi utilizado por $18,3 \%$, contudo, um percentual de 26,7\% utilizou em grande quantidade essa estratégia, que está relacionada aos esforços da pessoa em buscar o controle das emoçóes frente aos estímulos estressantes. Nesse sentido, é possível observar que o enfermeiro tenta controlar suas emoçóes, guardando seus sentimentos para si mesmo. Ter autocontrole denota, também, não fazer nada apressadamente ou seguir um primeiro impulso ${ }^{18}$. Resultado parecido foi encontrado ao avaliar as estratégias de coping em idosos institucionalizados ${ }^{23}$ e também em uma pesquisa com o uso de estratégias de coping na adolescência ${ }^{10}$.

O suporte social foi a terceira estratégia mais utilizada (Tabela 1). No total, 40,3\% utilizaram essa estratégia e $15,3 \%$ não a usaram. Essa estratégia, bastante utilizada pelos sujeitos, está relacionada ao apoio encontrado nas pessoas e no ambiente, sendo esse um fator psicossocial positivo, que pode ajudar o enfermeiro a lidar com o efeito indesejado do estresse, ao manifestar uma resposta apropriada à situação. A estratégia suporte social apresenta três diferentes aspectos. O primeiro deles refere-se à procura de apoio social para encontrar soluçôes; o segundo é o apoio emocional em amigos e familiares; e o terceiro é o apoio de outros profissionais ${ }^{22}$. Ela pode apresentar-se tanto centrada na emoção, em caso de suporte emocional, quanto no problema, na busca de suporte informativo. Vale ressaltar que o suporte socioemocional para os sujeitos desta pesquisa se refere predominantemente aos familiares, amigos e colegas profissionais, sendo declarado nos três casos um percentual de $83,3 \%$. Tais achados podem ser contemplados em outras pesquisas ${ }^{20,21,24}$.

Ao utilizar a estratégia de aceitação de responsabilidade (Tabela 1), um percentual de 38,1\% enquadra-se na classificação bastante utilizada e 17,9\%, na classificação não usada. Nesse caso, é possível observar que o enfermeiro aceita a realidade e engaja-se no processo de lidar com a situação estressante. Muitas vezes, o ele pode se sentir responsável por desencadear a situação estressante e, neste sentido, enfrenta sentimentos de autocrítica e repreensão. Como foi o caso desta pesquisa, em que cinco dos 12 enfermeiros compreenderam que a situação estressante foi provocada por eles; porém, esses sentimentos podem ter um efeito motivador, fato que estimula a enfrentar seus problemas de outra forma ${ }^{22}$, ao entender que as coisas devem ser diferentes na próxima vez. Isso foi visto em outras pesquisas, ao avaliar o coping em familiares de pacientes internados em Unidades de Tratamento Intensivo $(\mathrm{UTI})^{20}$.

Embora a fuga-esquiva tenha sido uma estratégia utilizada bastante $(37,5 \%)$, caracterizando-se o seu não uso em 25\%. Tais resultados (Tabela 1) demonstram que os enfermeiros ainda sentem dificuldades em se defrontar com a morte ou a possibilidade dela, pois o comportamento de fuga-esquiva consiste em fantasiar sobre possíveis soluçóes para o problema sem, no entanto, tomar atitudes para de fato modificá-las ${ }^{22}$, além de desejar que a situação acabe o mais rápido possível. Neste caso, pode-se descrevê-la como os esforços para escapar e/ou evitar o fator estressante. É possível observar em outros estudos a esquiva consciente da realidade da ameaça. Sendo uma estratégia centrada na emoção, que é caracterizada como uma tentativa de normalizar o impacto emocional do estresse no indivíduo, elas se tornam menos adaptativas do que a centrada no problema. Esse resultado foi visto em outro estudo que analisou os fatores estressantes e coping de enfermeiros responsáveis pelo tratamento de pacientes oncológicos ${ }^{17}$, além de outras pesquisas que mostram resultados semelhantes ${ }^{20-23}$.

Apesar de usarem fuga-esquiva, a estratégia de resolução de problemas (Tabela 1 ) foi a mais utilizada, sendo de $52,1 \%$ seu percentual, enquadrando-se na classificação: usada bastante. Observou-se que 35,4\% utilizaram essa estratégia em grande quantidade e apenas $6,3 \%$ não a usaram. Tal fato faz supor que esses sujeitos têm um planejamento adequado para lidar com os estressores, mesmo sentindo dificuldades em se defrontar com a morte de seus pacientes.

Esses dados mostram que, ao invés de anular ou afastar a situação estressante de seu cotidiano, o enfermeiro opta por tentar resolver seu problema, modificar suas atitudes, sendo capaz de lidar com as pressóes das pessoas e do ambiente ao seu redor, de modo a diminuir ou eliminar a fonte geradora de estresse ${ }^{22}$, ao se concentrar no que 
deveria se feito e recusar recuar e batalhar pelo que quer. Neste caso, supóe-se que ele pensará em um plano de ação, buscando concretizá-lo. Caracteriza-se em uma estratégia focalizada no problema são consideradas adaptativas, buscando remover ou abrandar a fonte estressora, ao levar em conta a realidade. Esses resultados foram encontrados em outros estudos ${ }^{20,23-24}$.

A reavaliação positiva (Tabela 1) foi a segunda estratégia mais usada; apesar de a estratégia de resolução de problemas ter sido utilizada por um maior número de participantes, a estratégia de reavaliação positiva foi empregada com mais frequência, sendo de $39,8 \%$ seu percentual, que enquadram-se na classificação: usada bastante e 13,9\% enquadraram-se na classificação não usada. Esse fato demonstra que os enfermeiros tendem a reestruturar o que aconteceu de forma a buscar, através dos aspectos positivos, resolverem a questão ou diminuir a carga afetiva direcionada à situação, sendo dirigida para o controle das emoçôes que estão relacionadas à tristeza como forma de reinterpretação, crescimento e mudança pessoal a partir da situação conflitante ${ }^{22}$, podendo apresentar dimensão religiosa, como foi visto no estudo em que dez dos 12 enfermeiros mostraram religiosidade diante do fato estressante e todos os enfermeiros que se sentiram culpados por desencadear a situação rezaram, o que foi observado em outras pesquisas ${ }^{10,20}$.

\section{CONCLUSÃO}

Os resultados desta pesquisa demonstram que os oito fatores propostos por Lazarus e Folkman (1984) foram evidenciados e que todos os sujeitos envolvidos na pesquisa utilizaram dessas estratégias o que corrobora resultados de pesquisas anteriores que revelam associação entre os diferentes estilos de enfrentamento, podendo apresentar ou náo o predomínio de uma determinada estratégia. Ao determinar as estratégias, as formas de enfrentamento mais utilizadas pelos enfermeiros foram resoluçáo de problemas, reavaliação positiva e suporte social, e a situação de estresse mais relatada foi a fase terminal, pois é nessa fase que ocorrem maiores situaçóes de estresse, tornando-se mais difícil e angustiante.

Esse uso pode significar que eles procuram um planejamento adequado para lidar com as situaçóes estressantes, ao utilizar formas positivas para solucionar os problemas, na tentativa de, através de planos e alternativas, diminuir a carga emocional, de modo a potencializar o enfrentamento das adversidades com o suporte social, recebendo qualquer informação, auxílio material ou afetivo oferecido por grupo ou pessoas através do envolvimento socioemocional. Com isso, permitiu-se concluir que o uso de mais de uma estratégia de coping é mais adaptativo do que usar apenas um tipo, visto que o indivíduo tem mais recursos para enfrentar a situação estressante.
A utilização em pouca quantidade de confronto, afastamento e fuga-esquiva pode ser também ressaltada, pois demonstra que os enfermeiros se preocupam e se envolvem, por não negarem suas responsabilidades no processo em que se encontram; mas, apesar de essas estratégias terem sido utilizadas com pouca frequência, a sua utilização demonstra que os enfermeiros ainda apresentam certas dificuldades, mas esses são compensados com o uso frequente de estratégias que ajudam positivamente no enfrentamento ao cuidar de pacientes oncológicos pediátricos.

Evidenciou-se, então, que o entendimento dessas estratégias pode proporcionar atitudes que irão ajudar nas necessidades do cuidador enfermeiro e assim amenizar o seu sofrimento e melhorar o processo de cuidar dos pacientes oncológicos pediátricos. Por isso, a necessidade de mais estudos dessas estratégias ajudará a melhorar a qualidade da assistência prestada ao paciente oncológico pediátrico e da família, através do bem-estar do enfermeiro envolvido nesse processo de cuidar, além de subsidiar o serviço no próprio hospital, pois esse poderá realizar uma melhor capacitação desses profissionais e assim melhorar $o$ atendimento e rendimento de cada um.

\section{CONTRIBUIÇÕES}

Maria Gizelda Gomes Lages e Maria Amélia de Oliveira Costa participaram da concepção, planejamento do projeto de pesquisa; obtenção, análise e interpretação dos dados; redação e revisão crítica. Thayson Rodrigues Lopes, Fráyla Cristinne Sousa de Amorim, Ari Pereira de Araujo Neto, Ianny Raquel Dantas Nascimento participaram da redação e revisão crítica. Charllyton Luis Sena da Costa participou da obtenção, análise e interpretação dos dados; redação e revisão crítica.

\section{Declaração de Conflito de Interesses: Nada a Declarar.}

\section{REFERÊNCIAS}

1. Gargiulo CA, Melo MCSC, Salimena AMO, Bara VMF, Souza IEO. Vivenciando o cotidiano do cuidado na percepção de enfermeiras oncológicas. Texto Contexto Enferm. 2007;16(4):696-702.

2. Instituto Nacional de Câncer (Brasil). Ações de enfermagem para o controle do câncer: uma proposta de integração ensino-serviço. 2a ed. Rio de Janeiro: INCA; 2002. $376 \mathrm{p}$.

3. Mutti CF, Paula CC, Souto MD. Assistência à saúde da criança com câncer na produção científica brasileira. Rev bras cancerol. 2010;56(1):71-83.

4. Silveira CS, Zago MMF. Pesquisa brasileira em enfermagem oncológica: uma revisão integrativa. Rev Latino-Am Enfermagem. 2006;14(4):614-9. 
5. Avellar LZ, Iglesias A, Valverde PF. Sofrimento psíquico em trabalhadores de enfermagem de uma unidade de oncologia. Psicol estud. 2007;12(3):475-81.

6. Silva LC. O sofrimento psicológico dos profissionais de saúde na atenção aos pacientes oncológicos. III Congresso Interamericano de Psicologia da Saúde; 2005 jun 9-11; São Paulo; BR. p. 96.

7. Margis R, Picon P, Cosner AF, Silveira RO. Relação entre estressores, estresse e ansiedade. Rev psiquiatr Rio Gd Sul. 2003;25(supl 1):65-74.

8. Sousa DM, Soares EO, Costa KMS, Pacífico ALC, Parente ACM. A vivência da enfermeira no processo de morte e morrer dos pacientes oncológicos. Texto Contexto Enferm. 2009;18(1):41-7.

9. Sanzovo CE, Coelho MEC. Estressores e estratégias de coping em uma amostra de psicólogos clínicos. Estud psicol (Campinas). 2007;24(2):227-38.

10. Kristensen CH, Schaefer LS, Busnello FB. Estratégias de coping e sintomas de stress na adolescência. Estud psicol (Campinas). 2010;27(1):21-30.

11. Diniz SS, Zanini DS. Relação entre fatores de personalidade e estratégias de coping em adolescentes. Psico-USF (Impr). 2010;15(1):71-80.

12. Antoniazzi AS, Dell'Aglio DD, Bandeira DR. O conceito de coping: uma revisão teórica. Estud psicol (Natal). 1998;3(2):273-94.

13. Guido LA, Bianchi ERF, Linch GFC. Coping entre enfermeiros de centro cirúrgico e recuperação anestésica. Rev Enferm UFPE On Line / J Nurs Ufpe online. 2009;3(4):35-7.

14. Savóia MG, Santana PR, Mejias NP. Adaptação do inventário de estratégias de coping de Folkman e Lazarus para o português. Psicol USP. 1996;7(1/2):183-201.

15. Barbosa RJ. Oncologia infantil e resiliência: um enfoque na saúde [trabalho de conclusão de curso]. São Paulo: Universidade Sagrado Coração, Escola de Psicologia; 2005.
16. Ferreira NMLA. A difícil convivência com o câncer: um estudo das emoções na enfermagem oncológica. Rev Esc Enferm USP. 1996;30(2):229-53.

17. Rodrigues AB, Chaves EC. Stressing factors and coping strategies used by oncology nurses. Rev Latino-Am Enfermagem. 2008;16(1):24-8.

18. Faria J. Paciente em longa internação: estratégias de enfrentamento dos familiares (coping) [trabalho de conclusão de curso]. São Paulo: Universidade de São Paulo, Instituto do Coração do Hospital das Clínicas da Faculdade de Medicina, Curso de Psicologia Clínica Hospitalar aplicado à Cardiologia; 2002.

19. Yamamoto MK. Internação prolongada: as estratégias de enfrentamento (coping) utilizadas por pacientes cardiopatas [trabalho de conclusão de curso]. São Paulo: Universidade de São Paulo, Instituto do Coração do Hospital das Clínicas da Faculdade de Medicina, Curso de Psicologia Clínica Hospitalar aplicado à Cardiologia; 2000.

20. Oliveira JF, Watanabe CE, Romano BW. Estratégias de enfrentamento ("COPING") dos familiares de pacientes internados em Unidade de terapia Intensiva. Rev Soc Cardiol Estado de São Paulo. 2007;17(supl 3A):4-9.

21. Lino CRG, Pacheco-Ferreira H. O impacto psicológico de um acidente químico ambiental com óleo diesel. Psicol estud. 2009;14(2):341-7.

22. Damiāo EBC, Rossato LM, Fabri LRO, Dias VC. Inventário de estratégias de enfrentamento: um referencial teórico. Rev Esc Enferm USP. 2009;43(spe 2):1199-1203.

23. Vivan AS, Argimon IIL. Estratégias de enfrentamento, dificuldades funcionais e fatores associados em idosos institucionalizados. Cad Saúde Pública. 2009;25(2):436-44.

24. Medeiros VCC, Peniche ACG. A influência da ansiedade nas estratégias de enfrentamento utilizadas no período pré-operatório. Rev Esc Enferm USP. 2006; 40(1):86-92. 


\section{Abstract}

Introduction: When facing the difficulties of taking care of children with cancer, coping strategies, which are understood as a set of behavioral responses of a person under a stressful situation and when trying to adapt themselves to the cause of the stress. Objective: To evaluate the Coping strategies nurses use towards pediatric oncologic patients; to determine the number of nurses who use Coping in their routine when taking care of pediatric oncological patients, and to identify the coping strategies and situations that nurses face when taking care of pediatric oncologic patients. Method: The research included quantitative, descriptive and exploratory settings. The data collection was done based on the application of the Coping Strategies Inventory, in the hospital established to the treatment of oncologic illness, Teresina-(PI) from March to September of the year 2010. The target population of this study was formed by all the nurses who work or have already worked in pediatric oncology of the abovementioned hospital, 20 out of 12 nurses agreed to participate. Results: The results demonstrate that all the interviewed population use Coping strategies and the most stressful situation was the terminal phase, and the strategy to face problem-solving situations is the most used by eleven nurses (93.8) and the least used one was the escape dodge, used by just nine nurses (75\%). Conclusion: The least use of negative strategies demonstrates that the nurses get worried and involved, and do not deny their responsibilities in the process they are in charge of. The interviewed nurses use mainly positive strategies, although they still turn to means that do not help them with the facing strategy.

Key words: Pediatric Nursing; Oncologic Nursing; Nurse-Patient Relations; Adaptation, Psychological; Child

\section{Resumen}

Introducción: En la dificultad de cuidar a los niños con cáncer se puede utilizar estrategias de enfrentamiento, entendidas como un conjunto de respuestas de conducta del individuo, ante la situación estresante y en la tentativa de adaptarse al evento de estrés. Objetivo: Evaluar las estrategias de coping de enfermeros frente al paciente oncológico pediátrico, determinar la cantidad de enfermeros que utilizan coping en el cotidiano al cuidar a pacientes oncológicos pediátricos e identificar las estrategias de coping y las situaciones de enfrentamiento de los enfermeros al cuidar de los, pacientes oncológicos pediátricos. Método: La investigación fue de naturaleza cuantitativa, descriptiva y exploratoria. La recogida de datos fue llevada a cabo a través de la aplicación del Inventario de Estrategias de Coping, en el hospital referenciado para el tratamiento de enfermedades oncológicas, Teresina-PI de marzo a septiembre de 2010. La población target del estudio estaba constituida en todos los enfermeros que ya trabajaban o trabajan en el área de oncología pediátrica del referido hospital, de un total de 20 enfermeros, 12 aceptaron participar. Resultados: Los resultados demuestran que la totalidad de la población encuestada utilizó estrategias de coping y la situación extrusora más citada fue la de la fase terminal, y la estrategia de enfrentamiento de resolución de problemas fue la más utilizada por 11 enfermeros $(93,8)$ y la menos utilizada fue la fuga-esquiva, utilizada por nueve enfermeros (75\%). Conclusión: La menor utilización de estrategias negativas demuestra que los enfermeros se preocupan, se comprometen, y no niegan sus responsabilidades en el proceso en que se encuentran. Los Enfermeros investigados utilizan predominantemente estrategias positivas, aunque aún recorran a medios que no ayudan en el enfrentamiento.

Palabras clave: Enfermería Pediátrica; Enfermería Oncológica; Relaciones Enfermero-Paciente; Adaptación Psicológica; Niño 